\title{
Mujeres que leen, escriben y calculan para participar en la economía familiar y local
}

\author{
Diana Solares, Facultad de Psicología, Universidad Autónoma de Querétaro (México) \\ David Block, Departamento de Investigaciones Educativas - Cinvestav (México)
}

Mujeres que leen, escriben y calculan para participar en la economía familiar y local

\section{Resumen}

Este artículo da cuenta de conocimientos matemáticos de dos grupos de mujeres indígenas en dos territorios mexicanos, con una escolaridad mínima o nula. Dichos conocimientos se pusieron de manifiesto en situaciones exploratorias diseñadas a partir de una caracterización de actividades cotidianas y laborales de las mujeres de ambos grupos. Damos cuenta también del cruce de miradas teóricas y analíticas que subyace a la metodología utilizada en el estudio. Nuestra finalidad es aportar elementos para el diseño de situaciones didácticas que, por una parte, consideren conocimientos construidos por las participantes en sus actividades sociales y culturales y, por otra parte, propicien la construcción progresiva de nuevos aprendizajes matemáticos.

Palabras clave. Educación matemática para adultas no alfabetizadas; prácticas socioculturales; exploración de conocimientos; diseños didácticos en matemáticas.

Women who read, write and calculate to participate in the family and local economy

\section{Abstract}

This article reports on the mathematical knowledge identified within two groups of indigenous women in two Mexican regions, with poor or no schooling. The body of mathematical knowledge is identified through exploratory situations whose design was based on a characterization of daily and working activities in which the women of each group participated. The crossing of theoretical and analytical lenses, which is put into discussion, allowed us to produce such an identification. Our purpose is to provide elements for the design of didactic situations that, on the one hand, draw on knowledge built by the participants in their social and cultural activities and, on the other hand, promote the progressive construction of newer mathematics learning.

Keywords. Mathematics education for non-literate women adults; sociocultural practices; knowledge exploration; mathematics didactical designs.

\section{Introducción y contexto}

En una comunidad indígena de Oaxaca, al sur de México, Julia, una mujer zapoteca de treinta años, quisiera saber cómo hace las cuentas el dueño de la tienda en la que compra, y cuánto recibirá de cambio (el sobrante) cada vez que ella paga con uno de los billetes que su esposo le envía desde algún campo de cultivo del norte del país. Julia también quiere aprender a "dar el cambio" porque podría vender en su pueblo, pero no se atreve por miedo a equivocarse. Además, quiere leer sin pedir ayuda a otros, quiere escribir su nombre y firmar con su puño y letra. De niña sólo cursó el primer grado de la escuela primaria, pues tuvo que migrar junto con su familia a los campos agrícolas del norte del país, donde ahora trabaja su esposo y varias familias de su comunidad.

En Yucatán, al sureste de México, otra mujer indígena aprende a cultivar hortalizas sin pesticidas y a criar gallinas. Elena, mujer maya de más de sesenta años, vende sus productos agrícolas a proveedores que los distribuyen a kilómetros de distancia. Dice no saber leer ni escribir porque "antes a las niñas no las dejaban ir a la escuela". Intentó 
aprender siendo mayor, pero era la única de su comunidad que asistía a las clases para adultos de manera continua por lo que se suspendió el servicio que daba el Instituto Nacional para la Educación de Adultos (INEA). Ahora aprende a usar la calculadora junto con otras productoras agrícolas, aunque prefiere hacer las cuentas "en su cabeza".

Julia y Elena forman parte de la población que se encuentra en "rezago educativo"; según el INEA, es la "población de 15 años y más que no sabe leer ni escribir y/o que no ha iniciado o concluido su educación primaria o secundaria" (INEA, 2016). De acuerdo con la Encuesta Intercensal de 2015, alrededor del 33\% de la población mexicana con 15 años o más de edad se encontraba en rezago educativo (28.630.060 habitantes), mientras que el $5.5 \%$ de esta última no sabía leer ni escribir (más de cuatro millones de personas, la mayoría mujeres).

¿Cómo resuelven esas personas necesidades cotidianas que requieren interpretar información numérica y hacer cuentas?, ¿qué es lo que saben hacer y qué quieren aprender?, ¿qué educación matemática se plantea para ellas? Estas son preguntas que intentamos contestar en proyectos que hemos venido desarrollando. En este artículo presentamos cómo nos hemos aproximado a conocimientos matemáticos de dos grupos de mujeres con ocupaciones distintas y rasgos comunes. Son mujeres indígenas con escolaridad mínima o nula que desean mayor autonomía económica y social. El primer grupo está formado por tres mujeres zapotecas de entre 30 y 40 años, de Oaxaca; el segundo grupo son ocho mujeres de una comunidad maya de Yucatán, de 25 a 60 años.

Las mujeres zapotecas pertenecen a familias jornaleras agrícolas migrantes, que para trabajar se trasladan fuera de la localidad donde está su hogar (en México hay unos 2.099.586 jornaleros agrícolas migrantes según la Encuesta Nacional de Jornaleros de 2009, citada en INEE, 2016). Entrevistamos a estas mujeres en su comunidad de origen, Coatecas Altas, lugar con un alto grado de marginación y constante migración a lo largo de generaciones, para laborar en campos agrícolas. Este grupo de mujeres manifestó su deseo de aprender a leer, a escribir y a hacer cuentas con la calculadora. Hicieron la petición en el marco de un proyecto de investigación sobre aprendizajes matemáticos que se desarrollaba con sus hijos en la escuela. Tras días de observar a la docente (la primera autora) trabajar matemáticas con sus hijos, las mujeres comentaron su dificultad para calcular cuánto les cobran en las tiendas de su comunidad y de los campos de cultivo a donde migran para trabajar; querían saber qué hacen con la calculadora los dueños de las tiendas para fijar el cobro y el dinero sobrante. Algunas expresaron que querían vender comida en su comunidad, por lo que querían aprender a cobrar y a dar el cambio.

Las mujeres mayas del grupo de Yucatán han recibido capacitación de parte de una asociación civil para aprender oficios y obtener ganancias. Este grupo de mujeres aprendió a cultivar hortalizas y a criar gallinas de manera "ecológica". El acercamiento que tuvimos con ellas fue en respuesta a la solicitud de la asociación civil para que se atendieran necesidades de aprendizaje de cálculo y de registro de cantidades, a fin de que las mujeres pudieran realizar mejor y con más autonomía sus tareas productivas.

Colaborar con el logro de los propósitos de cada grupo implicó esbozar rutas didácticas para la intervención educativa, sensibles a conocimientos previos y a necesidades específicas de las aprendices. Así surgió el reto de caracterizar actividades laborales y cotidianas en las que participan las mujeres de cada grupo, e identificar conocimientos en cada actividad. En lo que sigue resumimos las perspectivas teóricas y decisiones metodológicas que orientaron las exploraciones con cada grupo; describimos actividades específicas y conocimientos matemáticos que se movilizan. Acabamos con 
reflexiones sobre rutas didácticas que consideramos factibles y retos de la postura didáctica que asumimos ante la educación matemática para adultos.

\section{Antecedentes y marco teórico}

La opción de caracterizar las actividades cotidianas en las que participan las mujeres de cada grupo surge de planteamientos que comparten diversas perspectivas teóricas de la educación matemática y de otros campos. En Solares (2012a; 2012b, 2018), Solares y Block (2018) y Kalman y Solares (2018), hemos asumido estos planteamientos.

En América Latina se han desarrollado diversos estudios con el propósito de identificar conocimientos matemáticos de personas adultas no alfabetizadas (e.g. Ferreiro, Nemirovsky, Block, \& Dávila, 1987; Knijnik, 2015; Fuenlabrada \& Delprato, 2009; Broitman, 2012). En México, según Ávila (2014), los estudios sobre los saberes matemáticos de esta población se caracterizan por la diversidad teórica que va "desde la clara influencia piagetiana en los orígenes, hasta las praxeologías chevallardianas o la cognición situada que sustentan los trabajos en los últimos años" (p. 53). En esta diversidad teórica, vemos un elemento común. Dichos estudios reconocen la matemática como una práctica social y cultural, por lo que indagan los conocimientos de las personas adultas en actividades propias de su entorno; coinciden por tanto en que el conocimiento y la situación en la que este se genera están íntimamente relacionados (Solares, 2012b). Los análisis que presentamos se inscriben en ese conjunto de estudios latinoamericanos. Concebimos la matemática como práctica sociocultural y vemos la importancia de las circunstancias específicas -conceptualizadas de distinta manera, como contexto, actividad, situación o tarea, en diferentes teorías que aquí comentamos-en las que emergen conocimientos matemáticos y del significado de tales conocimientos.

\subsection{El carácter relativo del conocimiento matemático}

Un aspecto que nos permite asumir la concepción anterior es el reconocimiento del carácter relativo de los conocimientos matemáticos en función de la situación o de la institución en la que tengan lugar. Nuestros argumentos provienen de las teorías didácticas que han orientado buena parte de nuestra investigación: la Teoría de las Situaciones Didácticas (TSD) y la Teoría Antropológica de lo Didáctico (TAD).

La TSD plantea que un mismo conocimiento matemático puede tener distintos significados en función de la situación problemática de la que emerge, pues distintas situaciones pueden implicar diversos aspectos de un mismo conocimiento matemático, normalmente abstracto y general (Brousseau, 2000). Por ejemplo, la suma permite saber la cardinalidad de la unión de dos conjuntos disjuntos pero, además y particularmente para personas adultas no alfabetizadas, es la herramienta que permite abordar situaciones multiplicativas (iterando una cantidad, duplicando, contando el número de veces que una cantidad cabe en otra, etc.). La diversidad de procedimientos suele estar vinculada a características de las situaciones donde los procedimientos tienen lugar, como veremos con el grupo de mujeres mayas. Por su parte, la TAD también se está utilizando para indagar conocimientos matemáticos implícitos fuera de la escuela (Covián \& RomoVázquez, 2014; Solares, Solares, \& Padilla, 2016), en la línea de considerar la matemática como una actividad más del conjunto de actividades humanas cuya finalidad es resolver problemas mediante un trabajo de modelización, ya sea en prácticas escolares o no escolares. Dichas prácticas se construyen y difunden en instituciones específicas, por lo que adquieren características acordes con la institución que las alberga (Castela, 2008). De ahí que en torno a un mismo saber puedan haber varias prácticas matemáticas. 
Los planteamientos anteriores de la TSD y la TAD nos permiten subrayar el hecho de que las matemáticas, más allá de la escuela y de las demás instituciones formales reconocidas como productoras de saber matemático, tienen lugar en los conocimientos implícitos, personales, que producen las personas para resolver determinado tipo de problemas como obtener el cambio justo al hacer sus compras cotidianas.

\subsection{La matemática como práctica sociocultural ligada a actividades específicas}

Cuando realizamos nuestras primeras indagaciones de conocimientos matemáticos extra escolares, empleamos las teorías que orientaron nuestros estudios previos en didáctica. No obstante, desde un inicio vimos la necesidad de enriquecer la mirada con otras perspectivas que introdujeran de maneras más claras el componente del contexto. Acudimos entonces a dos referentes que se han desarrollado en nuestras instituciones: la Teoría de la Cognición en la Práctica, por un lado, y los estudios de New Literacy Practices, que nos aproximan a los de Numeracy As Social Practice.

La Teoría de la Cognición en la Práctica resalta la función de la actividad específica en el desempeño cognitivo de los sujetos. Algunos de los estudios sobre la actividad matemática en este marco analizan las formas en que las personas problematizan y resuelven situaciones cuando hacen las compras, siguen un plan de dieta o elaboran prendas de vestir (Lave, 1991, 2011). Esta teoría afirma que el papel social de las situaciones, de las interacciones entre personas y de los respectivos contextos influyen en las formas en que problematizamos y resolvemos determinadas situaciones; por ello, una misma persona difiere en sus actividades aritméticas en los distintos entornos. Estas ideas amplían el carácter situacional del conocimiento matemático, como vimos con la TSD y la TAD, considerando también el contexto en el que la situación se plantea, los motivos y las metas de los participantes, así como las interacciones entre ellos.

Asumimos que los contextos sociales, económicos y culturales son relevantes en la organización de nuestras acciones cuando pretendemos resolver un problema; de ahí que exploremos conocimientos matemáticos en situaciones y/o actividades propias del entorno de quienes los movilizan. Esta decisión metodológica y epistemológica está ligada a la concepción de las matemáticas como práctica sociocultural. Al respecto, en Solares (2012a, 2012b) se documenta que en los campos de cultivo a los que las familias migrantes llegan a trabajar, hay diversas actividades laborales y de la vida cotidiana en las que se produce e interpreta información numérica: el registro de la producción de cada trabajador, o el registro y pago de deudas que contraen las familias con las tiendas de los campos de cultivo. Se detectaron tensiones donde se manifestaban conocimientos matemáticos a los que acudían las personas para preservar sus intereses; por ejemplo, las familias trabajadoras hacían sus propios cálculos para que en la tienda no les cobraran de más al pagar por sus víveres; mientras que en el trabajo agrícola, las familias cuidaban que el encargado de registrar su producción no les anotaran de menos.

\subsection{La interpretación de números escritos como práctica sociocultural}

Para analizar las estrategias mediante las cuales las familias trabajadoras interactuaban con documentos de su entorno -tomando contexto, actividades específicas e intereses de los participantes- recurrimos a tres referentes teóricos: la Teoría de la Cognición en la Práctica, la TAD y la perspectiva llamada New Literacy Studies (NLS).

La TAD permitió analizar las técnicas de las familias para interactuar con números escritos en actividades laborales, así como sus discursos en torno a esas técnicas; para ello acudimos al "modelo praxeológico extendido de la TAD" (Castela \& VázquezRomo, 2011; Solares, 2012a; 2012b). Para el control de las deudas con la tienda fue más 
factible recurrir a la NLS dado que a diferencia del ámbito laboral, técnicas y discursos en torno a las actividades relativas a la compra de víveres eran menos explícitas.

Los estudios inscritos en NLS indagan prácticas situadas de lectura y escritura en diversos contextos (Street, 1984); cuestionan el carácter netamente instrumental de la lectura y de la escritura (Kalman \& Street, 2009); asumen la alfabetización (literacy) como práctica social históricamente situada, más allá de las habilidades para leer y escribir; y analizan las interpretaciones de los sujetos de los documentos, abarcando las interacciones entre personas a propósito de esos textos (Barton \& Hamilton, 1988). En ese marco, Kalman y Solares (2018) analizaron los procedimientos de una mujer para controlar sus deudas con la tienda de un campo de cultivo, las cuales eran registradas en un cuaderno por el dueño de la tienda. La mujer afirmaba no saber leer ni escribir. Las autoras centraron la atención en las condiciones sociales de producción (Blommaert, 2008) en las cuales se generaron las estrategias de registro, cálculo y control de deudas. Las condiciones de extrema desigualdad social de los campos de cultivo se materializan en interpretaciones de los registros numéricos y en estrategias para hacer cálculos aritméticos, particularmente cuando hay conflictos entre clientes y dueños. Los registros de deudas son portadores de esos conflictos e interacciones.

Dado que en la investigación actual los dos grupos de mujeres con los cuales trabajamos tienen una escolaridad mínima o nula, y tomando en cuenta que ambos grupos interactúan con información numérica escrita, nos resultó útil apoyarnos en la perspectiva de NLS para comprender las especificidades de algunas de las actividades en las que participan, así como las interacciones que tienen con portadores de información numérica (particularmente con el grupo de Oaxaca).

\subsection{Complementariedad con otros acercamientos teóricos}

La investigación sobre conocimientos matemáticos utilizados por las personas en su vida, más allá de conocimientos institucionales enseñados en la escuela, ha tenido un fuerte desarrollo en los últimos 30 años, y ha dado lugar a perspectivas teóricas diversas. Algunas de esas perspectivas han sido agrupadas en lo que se ha llamado Numeracy as Social Practice (NSP) (Yasukawa, Jackson, Kane, \& Coben, 2018). En Yasukawa et al. (2018), se identifican al menos cuatro enfoques teóricos de influencia: la Teoría de la Cognición Situada (nos hemos referido a ella como Cognición en la Práctica), la Teoría Histórico-Cultural de la Actividad, Literacy as Social Practice, y la Etnomatemática.

En nuestro trabajo asumimos las características comunes, esenciales, que subyacen a los acercamientos de la NSP, a saber, el interés por lo que las personas hacen con sus conocimientos matemáticos implícitos o explícitos en sus interacciones en contextos específicos, más que por los conocimientos y habilidades descontextualizados que se enseñan normalmente en la escuela. Coincidimos en que el foco en la práctica implica ver la actividad matemática (numeracy activity) como cultural, histórica y políticamente situada (Yasukawa et al., 2018). Además de estas coincidencias en lo general, en nuestro acercamiento teórico tienen un peso especial las dos teorías del ámbito de la enseñanza de matemáticas (TSD, TAD) poco referidas en los estudios sobre NSP.

\section{Decisiones metodológicas}

Para identificar las actividades en las que cada grupo pone en juego sus conocimientos matemáticos, realizamos un acercamiento de indagación etnográfica. Hicimos entrevistas semi-estructuradas planteadas colectivamente y otras individuales. Preguntamos a las mujeres en qué consistían las actividades en las que participaban, por 
qué se hacían de esa manera, qué instrumentos usaban, quiénes eran los participantes y cuáles sus propósitos. Les preguntamos, además, cuáles eran sus necesidades (qué se les dificultaba, qué querían aprender y por qué). En el caso de las mujeres mayas entrevistamos también a las "facilitadoras", quienes eran las encargadas de capacitar a las mujeres en las actividades productivas. También hubo observación de la actividad específica in situ. Esto fue posible particularmente con las mujeres mayas, con quienes pudimos presenciar una jornada en la que pesaron los productos que venderían, calcularon los montos a cobrar y registraron los productos vendidos y sus precios.

A partir de la información obtenida a través del acercamiento etnográfico, y con base en la TSD, más específicamente la Ingeniería Didáctica (Artigue, 1995), diseñamos situaciones problemáticas para profundizar en la indagación de los procedimientos de resolución de las mujeres de cada grupo. Este diseño conllevó un análisis previo de conocimientos matemáticos implicados en cada situación problemática, la anticipación de posibles procedimientos de resolución, así como la determinación de variables didácticas que podrían hacer más compleja o más accesible cada situación. Las situaciones problemáticas se plantearon sobre todo de manera grupal para advertir qué ayudas se daban entre participantes, cómo explicaban y/o justificaban sus procedimientos. Luego se analizaron los procedimientos de resolución. El diseño finalmente se puso a disposición de la asociación civil que trabaja con ellas.

\section{Las mujeres zapotecas y la interpretación y cálculo de deudas con la tienda}

Para las familias jornaleras migrantes, interpretar los registros de las deudas que adquieren con las tiendas de los campos de cultivo es relevante. Barrón (2012) ha documentado un sistema de crédito y de sobre precios en esas tiendas que deja a las familias trabajadoras en situación de "deudores cautivos". Eso también ocurre en comunidades originarias de las familias. Según lo narrado por las tres mujeres zapotecas de Oaxaca, en las tiendas de su comunidad padecen los altos precios, y la interpretación de las cuentas que hacen los dueños de las tiendas les es poco accesible.

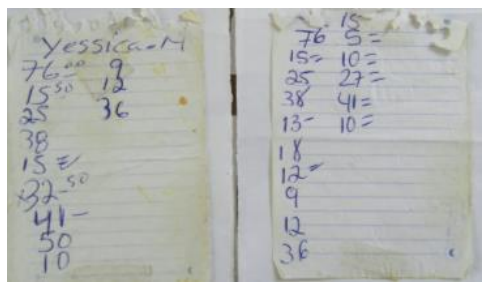

Figura 1. Registro de deudas con la tienda de un campo de cultivo

Con las mujeres zapotecas realizamos una exploración colectiva similar a las de estudios de la perspectiva NLS y que se han extendido a prácticas numéricas (Kalman $\&$ Street, 2009). Les presentamos documentos de un campo de cultivo (recibo de pago, registro del trabajo diario y página de una libreta de deudas de una tienda) y les planteamos preguntas como: ¿conocen algunos de estos documentos?, ¿cuál o cuáles han visto más?, ¿dónde los han visto?, ¿para qué se usan?, ¿quiénes los usan? Uno de los documentos más reconocidos fue el de las deudas de la tienda de la Figura 1. Las mujeres (cuyos nombres en las transcripciones son seudónimos) explicaron sus experiencias como clientes y deudoras de tiendas similares:

Julia: Ay, esas son de libretas.

Entrevistadora: ¿Libretas de qué? 
Julia:

Libreta para ir a pedir cosas fiadas ... porque ahí en la tienda dan una libretita donde pides las cosas que quieras y ya cuando llegue un día, viernes, sábado, ya vas y luego ya te dicen cuánto debes y ya lo pagas

Aun cuando algunas de las mujeres decían no saber leer ni escribir números, les preguntamos qué se pudo haber comprado de acuerdo con las cantidades registradas. En general no tuvieron gran dificultad para identificar los numerales, cuya interpretación parece apoyarse en sus experiencias de compra.
Entrevistadora: ... la primera que está aquí [76], ¿qué cantidad creen que sea?
Julia: ... Setenta y seis pesos.
Entrevistadora: $\quad$ Setenta y seis ¿Cómo qué se pudo haber pagado con setenta y seis pesos?
Bety: $\quad U y$, compró Maseca [harina para hacer tortillas].
Entrevistadora: ¿En cuánto está la Maseca?
Julia: $\quad$ La Maseca es lo más caro, la Maseca o la harina, porque un bulto nomás te cuesta ... Pues como setenta ... Sí, porque las cosas de allá son carísimas.

Julia y Bety manifestaron un conocimiento significativo de la numeración escrita; pudieron leer y escribir números hasta de tres cifras, aunque Julia tuvo dificultades que fue resolviendo en la interacción con Bety y la entrevistadora. La interpretación de las deudas llevó la conversación hacia los costos de la vida en el campo de cultivo, lo cual nos informó sobre el rango de las cantidades con las que suelen operar mentalmente.

Entrevistadora: ¿Y sí sale? ¿Para la renta, para la comida?

Julia: $\quad$ Muy apenitas. Como mi esposo trabajaba solo y tengo cuatro niños... Mi esposo ganaba mil quinientos, yo pagaba mil doscientos a la tienda y quedaban trescientos pesos ...

Para llevar el control de las deudas que adquieren en la tienda, las mujeres mencionaron dos recursos: apoyarse en quienes sí saben leer, escribir y hacer cuentas "a mano" o con calculadora (esposo o hijos), y apoyarse en su propia memoria.

Bety: $\quad$ Yo hago la cuenta pero me tardo. Veo que sí sale y mi esposo lo saca con lápiz.

Entrevistadora: Él lo hace con lápiz. ¿Y la calculadora no la usaban?

Bety: $\quad$ Sí la usaba también, pero él saca la cuenta más con lápiz y yo saco la cuenta más con memoria. Ya cuando tengo el total, entonces pongo el número y le digo a mi esposo y me dice ¿cómo sabes si no sabes leer ni escribir? Y le dije, pues no sé, pero sé más con la memoria.

Para profundizar en la exploración de procedimientos de cálculo y generar aprendizajes que ellas habían demandado, pedimos que se pusieran en el papel del dueño de la tienda cobrando deudas. El propósito fue colocarlas en una situación hipotética que las llevara a interpretar información escrita y a hacer cálculos aritméticos que "en la vida real" procuran evitar (como pedir al hijo más escolarizado que haga las compras). La entrevistadora actuó de "cliente", lo cual le permitió graduar la complejidad de los cálculos según el rango de precios, la cantidad de productos comprados y el billete con el que se pagaba. Proporcionamos monedas reales y billetes de imitación, papel, lápiz y calculadora, por si deseaban usarlos. La indagación de sus procedimientos y la intervención didáctica se desarrollaron de manera paralela, nutriéndose mutuamente. 
Tabla 1. Variantes de un problema aditivo

\begin{tabular}{|c|c|c|c|c|}
\hline Variante & Datos & Se busca & Operación & Problema \\
\hline 1 & $\begin{array}{l}\text { Billete }(\mathrm{B}) \\
\text { Precio }(\mathrm{P})\end{array}$ & Cambio (C) & $\begin{array}{l}\text { Resta } \\
\mathrm{C}=\mathrm{B}-\mathrm{P}\end{array}$ & $\begin{array}{l}\text { Cobrar } \$ 113 \text { de un billete de } \$ 200 . \\
\text { ¿Cuánto se dará de cambio? }\end{array}$ \\
\hline 2 & $\begin{array}{l}\text { Billete (B) } \\
\text { Cambio (C) }\end{array}$ & $\begin{array}{l}\text { Precio o lo que } \\
\text { se cobró }(\mathrm{P})\end{array}$ & $\begin{array}{l}\text { Resta } \\
\mathrm{P}=\mathrm{B}-\mathrm{C}\end{array}$ & $\begin{array}{l}\text { Pagué con un billete de } \$ 200 \text { y me dieron } \\
\text { de cambio } \$ 87 \text {. ¿Cuánto me cobraron? }\end{array}$ \\
\hline 3 & $\begin{array}{l}\text { Precio }(\mathrm{P}) \\
\text { Cambio }(\mathrm{C})\end{array}$ & $\begin{array}{l}\text { Valor del billete } \\
\text { de pago (B) }\end{array}$ & $\begin{array}{l}\text { Suma } \\
B=P+C\end{array}$ & $\begin{array}{l}\text { Compré un refresco de } \$ 17 \text { y me dieron } \\
\text { de cambio } \$ 33 \text {. ¿De cuánto era el billete? }\end{array}$ \\
\hline
\end{tabular}

Como en Solares y Block (2018), las mujeres mostraron conocimientos sobre lengua y números escritos y utilizaron procedimientos de cálculo mental. Se apoyaron entre ellas en español o en zapoteco, para interpretar números o calcular. Según los conocimientos que fueron manifestando a lo largo de las sesiones, planteamos variantes de un mismo problema aditivo. La Tabla 1 muestra la breve secuencia didáctica que promovía establecer relaciones entre datos e identificar la resta como operación que resuelve el problema. Destacamos los siguientes los procedimientos ante cada variante:

Variante 1. Es la situación más común que implica restar la cantidad que paga el comprador al valor del billete con el que paga; la diferencia es el cambio. Identificamos dos procedimientos: por complemento aditivo (que suelen usar dueños de tiendas de la comunidad), partiendo el sustraendo (cantidad cobrada) e ir agregando hasta llegar al minuendo (monto de billete entregado); o bien "quitando" o "contando al revés", partiendo del monto del billete entregado e ir quitando el monto del cobro mediante cálculos mentales. Se puede "feriar" o cambiar el billete por monedas de menor valor para después quitar el sustraendo; esto permite tener un mayor control de los cálculos.

Variante 2. Se calcula el monto de lo cobrado con el billete con el que se pagó y el cambio recibido como datos. Vemos de nuevo dos procedimientos: por complemento aditivo, haciendo descomposiciones, e.g. Irene hizo a un lado el 100 y operó con decenas y unidades (37), completó a 40 agregando tres unidades al 37 y luego agregó 60 para llegar al otro 100; o bien, "quitando" mediante calculadora con la tecla "menos".

Variante 3. Se promueve el procedimiento de restar mediante complemento aditivo (precio + cambio $=$ billete) en la situación original. Lo incluimos porque cuando se debía calcular el cambio, Bety, quien era muy hábil "quitando" o "contando al revés", tuvo dificultad para comprender el complemento aditivo usado por otras mujeres. No lograba pasar de la relación $\mathrm{B}-\mathrm{P}=\mathrm{C}$ a la relación aditiva $\mathrm{P}+\mathrm{C}=\mathrm{B}$. Resolvió la situación mediante cálculo mental y no supimos si, con esta intervención, comprendió el procedimiento de restar por complemento aditivo. Probablemente no porque se trató de una situación única (ella y su familia emigraron de la comunidad para trabajar nuevamente en campos de cultivo del norte de México).

\section{Las mujeres mayas y la venta de sus productos agrícolas}

El 7,4\% de la población del estado de Yucatán con 15 o más años de edad era analfabeta en 2015 (ver http://cuentame.inegi.org.mx). Una asociación civil de Yucatán ha fundado "casas comunitarias" donde se desarrollan proyectos educativos en cuatro comunidades indígenas. Una de las casas se ubica en Kimbilá, una comunidad con poco más de 600 habitantes. Un proyecto en Kimibilá es la instalación de granjas ecológicas en las casas de las mujeres participantes, donde se hacen tres tipos de actividad: recolección de miel de abejas meliponas, cultivo de hortalizas y cría de gallinas. Cada 
actividad se desarrolla en distintas fases que implican procedimientos sumamente específicos. Aquí describimos la fase del registro de la venta de productos de la granja.

Según explican las facilitadoras, las mujeres utilizan varios formatos para registrar la producción de su granja, la venta y las ganancias. Hay dos tipos de formato: los que se entregan a la asociación civil y el personal; este último se escribe en el cuaderno de cada mujer. Deben registrar qué consumen, qué venden a la comunidad y qué a la asociación, y cuánto ganan por mes, trimestre y año. El registro suele ser complejo ya que algunas mujeres afirman no saber leer ni escribir. Las facilitadoras usan imágenes de apoyo para quienes no leen y organizan al grupo por "niveles" para brindar ayuda. Los formatos han ido cambiando pues las facilitadoras procuran que las mujeres vayan aprendiendo con ellos; a veces se trata de aprendizajes sobre escritura y lectura, y otras veces sobre economía familiar "para que ellas mismas se percaten de cuánto ahorran". La facilitadora en Kimbilá apoya a 13 mujeres hablantes de maya. Afirma la dificultad de algunas para escribir números, mientras que otras hacen las cuentas con calculadora pero a veces no saben qué operación utilizar. La asociación civil nos solicitó que se atendieran esas necesidades educativas, por lo que entrevistamos a facilitadoras y revisamos registros de las mujeres. Con esa base, diseñamos una situación problemática (completar un formato de venta similar al que usan) para indagar conocimientos matemáticos (Figura 2). Esa indagación permitió identificar diversos conocimientos. Aquí resumimos procedimientos para resolver problemas multiplicativos.

\begin{tabular}{|c|c|c|c|c|}
\hline \multicolumn{5}{|c|}{ REGISTRO DE VENTAS -GRANJAS ECOLÓGICAS KI'KIL'JE } \\
\hline \multicolumn{5}{|l|}{ COMUNIDAD: } \\
\hline \multicolumn{3}{|l|}{ BENEFICIARIA: } & \multicolumn{2}{|l|}{ OLIO DE HOJA: } \\
\hline $\begin{array}{l}\text { FECHA DE COMPRA: } \\
28-11-2018\end{array}$ & $\begin{array}{l}\text { FECHA D } \\
12-12-18\end{array}$ & & PERSONA QUE PAGA: & \\
\hline PRODUCTO & UNIDAD & PRECIO & CANTIDAD COMPRADA & TOTAL \\
\hline HUEVO GRANDE & Pieza & $\$ 2.50$ & 46 & \\
\hline HUEVO CHICO & Pieza & $\$ 2.00$ & 16 & \\
\hline CILANTRO & Atado & $\$ 10$ & & $\$ 30$ \\
\hline RÁBANOS & Atado & $\$ 5$ & 6 & \\
\hline BERENJENA & Kilo & $\$ 20$ & & $\$ 15$ \\
\hline LECHUGA OREJONA & Atado & $\$ 10$ & 1 & \\
\hline LECHUGA LOCAL & Atado & $\$ 10$ & 2 & \\
\hline ACELGA & Atado & $\$ 8$ & & $\$ 32$ \\
\hline CEBOLLA & Kilo & $\$ 35$ & & $\$ 17.5$ \\
\hline IMPORTE TOTAL & & & & $\$ 301.50$ \\
\hline
\end{tabular}

Figura 2. Formato de venta para completar

Abordaremos ejemplos de resoluciones a problemas multiplicativos de cuarta proporcional, en los que se conocen tres valores y se debe calcular un cuarto valor; dichos valores pertenecen a dos magnitudes que son proporcionales, por ejemplo, número de huevos y precio de los huevos (ver una caracterización de estos tipos en Vergnaud, 1988). La suma fue la única operación que se utilizó, pero en circunstancias y con modalidades más complejas que en los problemas aditivos, para empezar, por la necesidad de considerar implícitamente propiedades básicas de la proporcionalidad. En los problemas que analizamos aquí se dio el valor unitario (e.g. los huevos cuestan $\$ 2.50$ cada uno). Desde el punto de vista de la estructura, estos son los casos más simples. Aun así, estos problemas implican la necesidad de manejar simultáneamente conteos de unidades de dos magnitudes (e.g. de huevos y de pesos) a partir de una relación que las vincula ("por cada huevo, 2.50 pesos"). Por otra parte, la dificultad de sumar muchas veces una misma cantidad lleva a hacer agrupamientos, algunos de uso local. 
Martha, la mujer de mayor edad del grupo y quien insistía en no saber leer ni escribir, durante la entrevista mostró conocimientos básicos. Para calcular cuánto debía pagar por 16 huevos si uno vale $\$ 2.00$, sumó iteradamente hasta tener la relación 5 huevos $-\$ 10$, la cual es cómoda para calcular, pues las sumas de 5 en 5 y, sobre todo, de 10 en 10 son fáciles. No obstante, se necesita llevar un control para saber cuántas veces hay que iterar los 5 huevos para llegar al múltiplo más cercano a 16. Esto no fue fácil para Martha.

Entrevistadora: Fueron dieciséis huevos chicos.

Martha: Dieciséis huevos chicos.

Entrevistadora: $\quad$ Y cada uno a dos, ¿cuánto será?

Martha: $\quad$ Cinco... diez, otro diez, veinte ¿y dieciséis?

Entrevistadora: Ya vamos diez [huevos].

Martha: [Contando] Dieciséis, diecisiete, dieciocho, ¿dieciséis?

Entrevistadora: Son dieciséis huevos, de diez huevos van veinte, ¿verdad?

Martha: $\quad$ Veinte.

Martha averiguó que por 10 huevos son $\$ 20$, pero otros 10 huevos es más de lo que necesita. Tuvo que regresar al conteo de uno en uno (un huevo, \$2), pero en ese cambio de agrupamiento (de 10 a 1), perdió el hilo de su procedimiento. Veamos ahora a Minerva, Gloria y Elena calculando el costo de 6 atados de rábanos. Estas mujeres usan la relación "por dos son diez pesos" e iterándola tres veces llegan sin dificultad a "por seis, son treinta pesos", esto es, $6 \times \$ 5=3(2 \times \$ 5)=3(\$ 10)=\$ 30$.

Entrevistadora: ... los rábanos a cinco pesos el atado, se compraron seis atados, ¿cuánto es?

Minerva: Treinta.

Entrevistadora: ¿Por qué treinta?

Minerva: $\quad$ Porque son cinco pesos.

Gloria: $\quad$ Son seis de a cinco.

Entrevistadora: $\quad$ Ajá, seis de a cinco.

Gloria: Dos son diez.

Entrevistadora: Dos son diez.

Gloria:

Si son seis, treinta.

Entrevistadora: $\quad$ Cuatro, veinte. Seis, treinta.

Finalmente, veamos los procedimientos de Elena. Se le planteó en una entrevista individual el problema de cuánto cobrar por 46 huevos de $\$ 2.50$ cada uno. Elena tuvo dificultad para hacer el cálculo tanto mentalmente como con calculadora (en proceso de aprender a usar). Para ayudar, la entrevistadora intercaló situaciones de estimación.

Entrevistadora: $\quad$... a ver, en su mente. Si yo le diera cincuenta pesos ... ¿Usted me los aceptaría, por esos cuarenta y seis huevos?

Elena: $\quad$ No.

Nos centramos en el tercer intento. Se le ofreció pluma y papel y Elena decidió usarlos para representar gráficamente los 46 huevos, en filas de 10 (Figura 3).

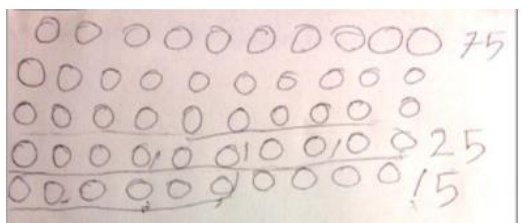

Figura 3. Representación de 46 huevos en filas de 10 
Con el apoyo de esta representación descompuso la cantidad en 30, 10 y 6 huevos. Sabía que 30 huevos corresponden a una "reja" (forma local de vender los huevos) y cuestan $\$ 75$; después calculó mentalmente para 10 huevos y para 6 .

Elena: Son diez, veinte, treinta, así, una reja, así [señalando los círculos].

Entrevistadora: $\quad$ Ah, una reja ... Ah, lo está haciendo por rejas.

Elena: Ajá, una reja, como los cuarenta y seis, agarro otros diez acá.

Entrevistadora: Sí.

Elena: Ahí tengo otros diez.

Entrevistadora: $\quad$ Sí.

Elena: Y agarro otros seis acá.

Para calcular para 10 huevos y para 6, iteró el par “dos huevos, cinco pesos". Así evitó calcular con decimales y trabajó con un sumando "cómodo". La sugerencia de la entrevistadora de anotar las tres cantidades conforme las fue obteniendo, le ayudó.

Elena: Son dos [huevos] cinco [pesos], diez [señala primeros 4 huevos, cuarta fila], quince [pesos] [señala 6 huevos], veinte, veinticinco [pesos por 10 huevos].

En el procedimiento para multiplicar vemos el uso sistemático de una relación entre cantidades de huevos y de pesos, cómoda para operar: 2 huevos, 5 pesos. También vemos el recurso a una relación conocida con un agrupamiento local: la reja. Se procede según la propiedad de la proporcionalidad para la cual si una cantidad aumenta $n$ veces, la que le corresponde aumenta n veces (conservación de las razones). Además, entra en juego la propiedad de que a la suma de dos cantidades de una magnitud, le corresponde la suma de las cantidades asociadas en la otra magnitud. Un esquema podría ser:
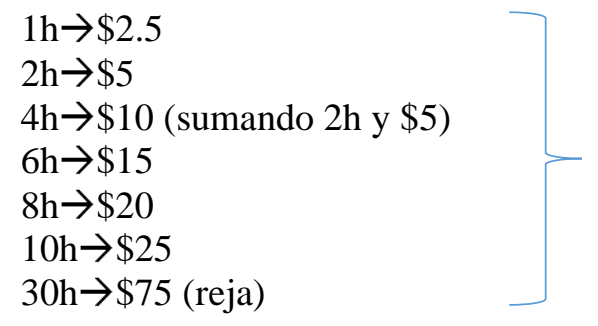

$$
\begin{array}{ll}
46 \text { huevos }= & 30+10+6 \\
\text { Costo }= & \$ 75+\$ 25+\$ 15
\end{array}
$$

\section{Consideraciones para la enseñanza}

Del trabajo con los dos grupos de mujeres, surgen consideraciones para la enseñanza. Con el primer grupo, hemos procurado que el diseño de las situaciones didácticas responda con una perspectiva amplia sobre lo que significa "saber hacer las cuentas". Más específicamente, tomamos en cuenta los siguientes aspectos en procesos de diseño e intervención didáctica: 1) Plantear variantes de un problema para evidenciar distintos procedimientos de resolución de las participantes y favorecer su mejora; 2) Propiciar el intercambio y la ayuda entre pares, particularmente cuando se pretende que las participantes formulen sus procedimientos; 3) Usar recursos (como dinero y calculadora) que permitan evidenciar relaciones numéricas y procedimientos a las participantes; 4) Recurrir a la calculadora para que las participantes descubran que un problema resuelto por complemento aditivo, también se resuelve con resta.

Las mujeres entrevistadas abordaron los problemas multiplicativos utilizando la relación "por cada unidad de una magnitud, se consideran $\mathrm{n}$ unidades de otra", la cual dio lugar a relaciones equivalentes que permiten hacer más eficiente el conteo: "por dos unidades, dos n", "por cada cinco unidades, cinco n", y, en general, "por cada m 
unidades, eme n". La utilización de estas relaciones constituye una base a partir de la cual se puede desarrollar el conocimiento de la multiplicación.

Las situaciones diseñadas para identificar conocimientos pueden ser fecundas para propiciar aprendizajes. Esto fue evidente con las mujeres zapotecas, con quienes el contacto fue mayor, mientras que con las mujeres mayas sólo fue posible hacer un diseño de intervención tras implementar las situaciones problemáticas y analizar los resultados. Su implementación podría contemplar estas metas: 1) Si se cuenta con la escritura de números, apoyar el control de relaciones del tipo "por cada $\mathrm{x}, \mathrm{y}$ ", mediante el uso de tablas de dos columnas; 2) Resolver problemas de comparación de razones con números naturales en distintos contextos; e.g. ¿dónde es más barato el huevo, donde lo venden a 5 por $\$ 10$, o donde lo venden 2 por $\$ 5$ ? (Block, 2006); 3) Explicitar situaciones multiplicativas y distinguirlas de las aditivas; e.g. para resolver cuánto hay que pagar por 5 huevos de $\$ 2$ cada uno, se puede hacer la operación $5 \times 2$, pero para resolver cuánto pagar en total si se compraron dos productos, de $\$ 35$ y de $\$ 24$, no es pertinente $35 \times 24$; 4) Introducir la calculadora como una forma más de calcular; 5) Introducir el contexto del cálculo de superficies de rectángulos dado que posibilita realizar agrupamientos gráficos visuales; 6) Ampliar el campo de problemas incluyendo casos de cuarta proporcional sin dar el valor unitario (Block, Mendoza y Ramírez, 2010); al mismo tiempo, los problemas pueden implicar medidas no enteras y cambios de unidad.

\section{Reflexiones finales}

Orientamos las dos experiencias a fin de contribuir al conocimiento sobre los saberes matemáticos de adultos con una escolaridad mínima o nula, y aportar elementos para el diseño de situaciones didácticas pertinentes para una población específica. Con respecto a la pertinencia, nuestra metodología consta de un proceso de indagación de los saberes de los adultos mediante el planteamiento de situaciones diseñadas a partir de: a) un conocimiento de su contexto y de las actividades que efectivamente realizan; b) un conocimiento didáctico en torno a cada noción implicada en dichas actividades y a las maneras en que se manifiesta en una diversidad de problemas. Esta indagación visibiliza la riqueza de conocimientos de los adultos, valiosa desde el punto de vista de nuestra comprensión de la cognición humana, y para el desarrollo de proyectos didácticos en los que dichos conocimientos deberían constituir una base imprescindible.

Desde otras perspectivas teóricas se ha realizado un importante trabajo de detección de contextos, tareas, actividades del entorno de estudiantes, que aportan problemáticas matemáticamente abordables, con vistas al aprovechamiento para la enseñanza (ver los conceptos de Culturally Relevant Pedagogy, Ladson-Billings, 1995; y de Funds of Knowledge Approach, González, Moll \& Amanti, 2005). Nosotros no estamos en el punto de estudiar propuestas para la escuela, sino en el de identificar conocimientos matemáticos en actividades específicas y esbozar posibles rutas didácticas. Para ello hemos explorado vías teóricas que nos han permitido avanzar. En la continuación de nuestro trabajo, nos interesa trazar un mapa de alternativas teóricas que se están generando, y de esta manera enriquecer y reorganizar el propio marco de referencia.

El proceso de indagación de conocimientos de las participantes y el proceso de intervención didáctica tienen fronteras borrosas. Las situaciones diseñadas para identificar conocimientos devenían en situaciones para la enseñanza y viceversa: las primeras, cuando se planteaban mediante situaciones abiertas que implicaban a los conocimientos, solían dar lugar a aprendizajes; las segundas, diseñadas y planteadas para enseñar, permitían identificar conocimientos, sobre todo en fases iniciales. Los 
enriquecimientos mutuos de ambos procesos nos llevan a matizar distinciones tajantes que suelen hacerse, que reflejan una de las tensiones en el rol del investigador (¿intervenir para conocer o para enseñar?) (Broitman, 2012). Procuramos aprovechar dicha tensión para los propósitos de la investigación y del diseño didáctico. Con respecto a los proyectos didácticos, algunas rutas a explorar son: a) potenciar, mediante escritura y otras representaciones gráficas, conocimientos de los adultos; b) considerar dos funciones de la calculadora, una para potenciar lo que saben hacer y otra para relacionar procedimientos que tienen con nociones que aún no tienen; e.g. asociar las sumas para hallar un complemento aditivo, con la resta; las sumas iteradas con la multiplicación; o las restas iteradas con la división; c) continuar la exploración de situaciones y contextos.

Hay limitaciones para desarrollar todos y cada uno de los aspectos didácticos señalados, debido en buena medida a condiciones de precariedad económica en personas adultas no alfabetizadas. Las mujeres participantes son responsables de distintas actividades con su familia y comunidad, por lo que les suele ser difícil disponer de tiempo. Por tanto, es difícil incorporar el desarrollo de proyectos didácticos en esa cotidianidad. Al mismo tiempo, nuestra limitación de no saber maya ni zapoteco, nos impedía comprender las ayudas mutuas que se daban entre las mujeres en sus lenguas, lo cual fue una oportunidad para ellas: la tarea de traductora implicaba, para quien la llevaba a cabo, formular, reelaborar, hacer explícitas relaciones numéricas o de otro tipo en su propia lengua, lo cual tiene un alto impacto en términos cognitivos, culturales y sociales tal como ha probado la investigación sobre lengua y educación matemática.

\section{Agradecimientos}

A Fundación Zorro Rojo A.C. y a Fundación Legorreta Hernández, por su invaluable apoyo y acompañamiento para la realización del trabajo de campo en Yucatán. A Natalia García Hess, Samanta Guerrero Neri, Berenice Cabrera Camargo y Paola Chica Hernández, por sus excelentes transcripciones.

\section{Referencias}

Artigue, M. (1995). Ingeniería Didáctica. En M. Artigue, R. Douady, L. Moreno, \& P. Gómez (Eds.), Ingeniería didáctica en educación matemática: Un esquema para la investigación y la innovación en la enseñanza y el aprendizaje de las matemáticas (pp. 33-60). Bogotá, Colombia: Grupo Editorial Iberoamericana.

Ávila, A. (2014). Del saber de la experiencia al saber en la experiencia: 25 años de investigación sobre saberes matemáticos y escolarización tardía en México. Educación Matemática, 26, n. extraordinario, 52-72.

Barrón, A. (17 de marzo de 2012). Dónde están y cómo están los jornaleros agrícolas. La Jornada del Campo. Recuperado el 30 de enero de 2021 de http://www.jornada. unam.mx/2012/03/17/cam-agricolas.html

Barton, D., \& Hamilton, M. (1998). Local literacies. Reading and writing in one community. Londres, Inglaterra: Routledge.

Block, D. (2006). Se cambian fichas por estampas. Un estudio didáctico sobre la noción de razón "múltiplo" y su vinculación con la multiplicación de números naturales. Educación Matemática, 18(2), 5-36.

Block, D., Mendoza, T., \& Ramírez, M. (2010). ¿Al doble le toca el doble? La enseñanza de la proporcionalidad en la educación básica. Colección "Somos Maestr@s” de la Serie “Enseñar y Aprender”. Ciudad de México, México: SM. 
Blommaert, J. (2008). Grassroots literacy: Writing, identity and voice in Central Africa. Londres, Inglaterra: Routledge.

Broitman, C. (2012). Adultos que inician la escolaridad: Sus conocimientos aritméticos y la relación que establecen con el saber y con las matemáticas. Trabajo de Tesis Doctoral. Buenos Aires, Argentina: Universidad Nacional de La Plata.

Brousseau, G. (2000). Educación y didáctica de las matemáticas. Educación Matemática, 12(1), 5-38.

Castela, C. (2008). Travailler avec, travailler sur la notion de praxéologie mathématique pour décrire les besoins d'apprentissage ignorés par les institutions d'enseignement. Recherches en Didactique des Mathématiques, 28(2), 135-182.

Castela, C., \& Romo-Vázquez, A. (2011). Des mathématiques á l'automatique: Étude des effets de transposition sur la transformée de Laplace dans la formation des ingénieurs. Recherches en Didactique des Mathématiques, 31(1), 79-130.

Covián, O., \& Romo-Vázquez, A. (2014). Las matemáticas en la construcción: La vivienda maya, el levantamiento y trazo topográfico. Bolema: Boletim de Educação Matemática, 28(48), 128-148.

Ferreiro, E., Fuenlabrada, I., Nemirovsky, M., Block, D., \& Dávila, M. (1987) Conceptualizaciones matemáticas en adultos no alfabetizados. Ciudad de México, México: DIE-CINVESTAV.

Fuenlabrada, I., \& Delprato, F. (2009). Prácticas matemáticas en organizaciones productivas de mujeres con baja escolaridad: construir una mirada que cimiente propuestas de enseñanza. En J. Kalman, \& B. Street (Eds.). Lectura, escritura y matemáticas como prácticas sociales. Diálogos con América Latina (pp. 242-263). Ciudad de México, México. CREFAL/Siglo XXI Editores.

González, N., Moll, L., \& Amanti, C. (Eds.) (2005). Funds of knowledge: Theorizing practice in households, communities, and classrooms. Mahwah, NJ: Lawrence Erlbaum Associates.

Instituto Nacional para la Evaluación de la Educación (2016). Directrices para mejorar la atención educativa de niñas, niños y adolescente de familias de jornaleros agrícolas migrantes. Ciudad de México, México: Autor.

Kalman, J., \& Street, B. (Eds.) (2009). Lectura, escritura y matemáticas como prácticas sociales. Diálogos con América Latina. Ciudad de México, México: Siglo XXI.

Kalman, J., \& Solares, D. (2018). Tear it out and rip it up or you might get charged again': Paying debts at the Company store in a farm workers' camp in Mexico. En K. Yasukawa, A. Rogers, K. Jackson, \& B. Street (Eds.), Numeracy as social practice (pp. 59-75). Nueva York: Routledge.

Knijnik, G. (2015). Etnomatemática y problemas auténticos en la formación del profesorado de matemáticas. En N. Planas (Ed.), Avances y realidades en la educación matemática (pp. 153-169). Barcelona: Graó.

Ladson-Billings, G. (1995). But that's just good teaching! The case for culturally relevant pedagogy. Theory Into Practice, 34(3), 159-165.

Lave, J. (1991). La cognición en la práctica. Barcelona: Paidós.

Lave, J. (2011). Apprenticeship in critical ethnographic practice. Chicago, IL: University Press. 
Solares, D. (2012a). Conocimientos matemáticos de niños y niñas jornaleros agrícolas migrantes. Trabajo de Tesis Doctoral. Ciudad de México: Departamento de Investigaciones Educativas-Cinvestav.

Solares, D. (2012b). Conocimientos matemáticos en situaciones extraescolares. Análisis de un caso en el contexto de los niños y niñas jornaleros migrantes. Educación Matemática, 24(1), 5-33.

Solares, D. (2018). Registros numéricos en un campo de cultivo. Escrituras en tensión en un ambiente laboral. PNA, 12(4), 209-228.

Solares, D., Solares, A., \& Padilla, E. (2016). La enseñanza de las matemáticas más allá de los salones de clase. Análisis de actividades laborales urbanas y rurales. Educación Matemática, 28(1), 69-98.

Solares, D., \& Block, D. (2018). ¿Cómo me están dando el cambio?... Así no les entiendo. Revista Decisio. Saberes para la Acción en Educación de Adultos, 51, $63-$ 69.

Street, B. V. (1984). Literacy in theory and practice. Cambridge, Inglaterra: University Press.

Vergnaud, G. (1988). Multiplicative structures. En. J. Hiebert, \& M. Behr (Eds), Number concepts and operations in the middle grades (Vol. 2, pp. 141-161). Hillsdale, NJ: Erlbaum.

Yasukawa, K., Jackson, K., Kane, P., Coben, D. (2018). Mapping the terrain of social practice perspectives of numeracy. En K. Yasukawa, A. Rogers, K. Jackson, \& B. Street (Eds), Numeracy as social practice: Global and local perspectives (pp. 3-17). Nueva York: Routledge.

\section{Referencias de los autores}

Diana Solares, Facultad de Psicología, Universidad Autónoma de Querétaro (México). violetasolares@gmail.com

David Block, Departamento de Investigaciones Educativas -Cinvestav (México). davidblock54@gmail.com 


\title{
Women who read, write and calculate to participate in the family and local economy
}

\author{
Diana Solares, Facultad de Psicología, Universidad Autónoma de Querétaro \\ David Block, Departamento de Investigaciones Educativas -Cinvestav
}

This article reports on the mathematical knowledge identified within two groups of indigenous women with poor or no schooling in two rural regions of Mexico. The body of mathematical knowledge is identified through exploratory situations whose design was based on a characterization of daily activities in which the women of each group participate. The crossing of theoretical and analytical lenses, which is put into discussion, allowed us to produce such an identification, but also to develop design work for the creation of didactical proposals. Our purpose is to provide elements for the design of didactic situations that, on the one hand, draw on knowledge built by the participants in their social and cultural activities and, on the other hand, promote the construction of new mathematics learning for them. The exploratory situations were designed considering elements of the situations that the women adults face, which were previously identified in the fieldwork through in situ observations and group and individual interviews. Also, for the design process, but especially for the analysis of the mathematical knowledge put into action, we relied on contributions from the didactics of mathematics on the specific notions mobilized in the practices observed. In the article, we present some examples of the productions that we collected and analyzed, which involve interpretation and writing of numbers, as well as knowledge about additive and multiplicative situations with a function in the daily and work places. The resolutions were almost always oral, sometimes including the use of graphic representations and of calculators. Overall, these resolutions and their related procedures highlighted the amount of mathematical resources deployed by the participants. Based on the fieldwork and findings, we come to outline possible routes of content-specific didactic work out of the situations that covered both exploration and teaching purposes In this way, our study contributes to the domain of mathematics education research with the focus on the mathematical knowledge and learning of groups of people in their communities. 